realisation has gradually emerged from a carefully directed research programme extending over 16 years by K. S. Warren of the Case Western Reserve School of Medicine and a number of collaborators. Warren has summarised the evidence that granuloma formation results from a delayed hypersensitivity reaction (Trans. roy. Soc. Trop. Med. Hyg., 66, 417; 1972) and has since extended these findings with the collaboration of D. L. Boros who originally joined him as a postdoctoral research worker. On page 224 of this issue they describe how they, and Pelley, have isolated granulomas in vitro and have extracted from antigen stimulated granulomas a substance that inhibits the migration of macrophages and which they identify as a lymphokine. In another paper Boros and Warren (Immunology, 24, 511; 1973) describe how the intravenous injection of bentonite particles impregnated with the antigen elicits granuloma formation in the lungs of sensitised mice.

The antigen is a soluble substance released from the egg. After the egg is laid embryonation occurs and this is accompanied by the liberation of enzymes and soluble antigens (which may be enzymes). The egg acts as a focus for the granuloma. Macrophages enter the immediate vicinity of the egg and come under the influence of the lymphokine and are unable to move away. In most bacterial and fungal infections this kind of local reaction isolates the invading organisms which are then phagocytosed by the macrophages. In schistosomiasis, however, a granuloma 100 times the size of the egg is formed and this is gradually replaced by fibrous scar tissue. Boros and Warren have also found that bentonite particles impregnated with mycobacterial and histoplasma antigens induce granulomas in animals sensitised with homologous antigens but not heterologous ones.

It is clear from these experiments that the pathology of schistosomiasis is not unique and comparable situations probably exist in a number of parasitic infections. The existence of non-protective delayed hypersensitivity states in parasitic diseases makes vaccination attempts potentially dangerous procedures which might even lead to immunological enhancement. The World Health Organisation (Tech. Rep. Ser., No. 519, Cell Mediated Immunity and Resistance to Infection; 1973) is fully aware of this problem and as more information about immunity to parasitic diseases accumulates the possibilities of developing useful methods of immunisation fade further and further into the distance.

F. E. G. C.

\section{CONTRACEPTION \\ How Sufe is Sufe?}

from a Correspondent

How safe is the 'safe' period as a means of contraception? This will depend among other things upon how long the ovum and sperm can exist in the reproductive tract without loss of the ability for fertilisation and how fixed in the menstrual cycle is the time of ovulation. Can ovulation in normally menstruating women occur at times other than at about mid-cycle? Can it be induced, or can spontaneous ovulation be hastened, by coitus? Although coitusinduced ovulation occurs in many species it is not usually considered to be a phenomenon in humans.

Jöchle (Contraception, 7, 523; 1973) has now reviewed the evidence relating to this question. The length of the luteal phase of the cycle seems to be fairly fixed at about 14 days; variations in menstrual cycle length usually result therefore from variability of the follicular phase. It is known that hypothalamic-pituitary-ovarian function can be modified by environmental change resulting in inhibition of ovulation and a consequent change of menstrual pattern or the complete absence of menstruation (psychogenic amenorrhoea).

The possibility exists therefore that other environmental changes might stimulate pituitary gonadotrophin secretion and produce ovulation. That ovulation can occur at times other than mid-cycle has been suggested by examination at autopsy of ovaries and uterus and calculation of the time interval in which ovulation must have occurred. When this interval was related to the time of menstruation of the previous cycle the results suggestcd that ovulation could occur at any time in the cycle. Similar observations made at surgical operations also suggested that ovulation could occur on any day of the first half of the cycle.

Evidence in support of coitus-induced ovulation was obtained from two sources. (1) Conceptions resulting from rape, using data only from patients for whom reliable information could be

\title{
Haemin Selectively Stimulates $\alpha$ Globin Synthesis
}

THE study of the role of haemin in protein synthesis is interesting because it provides evidence that some regulation takes place at the level of translation. When haemin is added to a cellfree system containing globin messenger RNA globin synthesis is stimulated, but so too is the synthesis of endogenous proteins. By contrast, when its effect is studied in an in vitro system (for example, when globin mRNA is injected into Xenopus oocytes), only the synthesis of globin is increased.

In Nature New Biology next Wednesday (November 28) Giglioni et al. describe some experiments designed to help elucidate the function of haemin in globin synthesis. Using the oocyte system they have found that in conditions of rabbit globin mRNA saturation there is about four times more $\beta$ globin than $\alpha$ globin synthesised. By increasing the amount of haemin added to the system $\alpha$ globin synthesisation is proportionally increased until a haemin concentration of $30 \mu \mathrm{M}$ when the ratio remains constant with $\alpha$ globin nearly $40 \%$ of the total globin synthesised in the system.

Endogenous protein synthesis is unaffected even at high haemin concentrations. At subsaturating levels of mRNA an $\alpha: \beta$ globin ratio of $1: 1$ can be achieved by injecting haemin into the oocytes, and if globin mRNA rich in $\alpha$ messenger is used more $\alpha$ than $\beta$ is synthesised.
From these results alone, the possibility cannot be excluded that haemin affects the recovery of the globin chains, after their release from the ribosome, rather than their synthesis. The results of control experiments, however, make the first suggestion extremely unlikely. Control experiments also exclude the possibility that the low $\alpha$ to $\beta$ ratio, in the absence of haemin, is due to a delay in the release of $\alpha$ chains relative to $\beta$ chains.

It seems clear that in this system haemin controls the synthesis of $\alpha$ but not $\beta$ mRNA. The authors suggest that the mRNAs for $\alpha$ and $\beta$ globin compete for some component necessary for their translation (but not that of endogenous mRNAs), and that the effect of haemin is restricted as soon as mRNA concentration becomes saturating.

It is difficult to know how far these results can be extrapolated to explain the action of haemin in intact reticulocytes. If reticulocyte-rich blood is incubated in the presence of lead the synthesis of haemin is inhibited and this is followed by a $90 \%$ reduction in globin synthesis. The absence of haemin in the oocyte system does not have such a depressing effect. In haemin-free reticulocytes, however, the $\alpha$ globin synthesis is inhibited more than that of $\beta$ globin so that in this important aspect the oocyte system and reticulocytes are the same. 